\title{
Random quadruple coincidence points theorems for sequence of random mappings and application
}

Xiaofang Yan, Chuanxi Zhu*, Zhaoqi Wu

Department of Mathematics, Nanchang University, Nanchang, 330031, P. R. China.

Communicated by C. Park

\begin{abstract}
In this paper, we give the definitions of compatibility and weakly reciprocally continuity for sequence of random mappings $T_{i}$ and a random self-mapping $g$. Further, using these definitions we establish quadruple random coincidence and quadruple random fixed point results by applying the concept of an $\alpha$-series for sequence of mappings, introduced by Sihag et al. [V. Sihag, R. K. Vats, C. Vetro, Quaest. Math., 37 (2014), 1-6], in the setting of partially ordered metric spaces. Our results are some random versions and extensions of results relating to triple fixed points theorems by R. K. Vats et al. [R. K. Vats, K. Tas, V. Sihag, A. Kumar, J. Inequal. Appl., 2014 (2014), 12 pages], we also give some examples to illustrate our results. (C)2016 All rights reserved.
\end{abstract}

Keywords: Random quadruple coincidence point, quadruple random fixed point, $\alpha$-series, partially ordered metric space.

2010 MSC: 47H10, 60H25.

\section{Introduction and preliminaries}

Random nonlinear analysis is an important mathematical discipline which is mainly concerned with the study of random nonlinear operators and their properties and is needed for the study of various classes of random equations. Random fixed point theorems are stochastic generalizations of classical fixed point theorems. Random fixed point theorems for contraction mappings on separable complete metric spaces have been proved by several authors (see Refs. [6], 7], [8], [9], [11], [13], [14], [15], [20], [24], [25], [26]).

\footnotetext{
${ }^{*}$ Corresponding author

Email addresses: xiaoxiaoyan_green@163.com (Xiaofang Yan), chuanxizhu@126.com (Chuanxi Zhu ), wuzhaoqi_conquer@163.com (Zhaoqi Wu)
} 
Bhaskar and Lakshmikantham [3] introduced the notion of a coupled fixed point and proved some coupled fixed point theorems for mappings satisfying a mixed monotone property. They discussed the problem of uniqueness of coupled fixed point and applied their theorems to problems of existence and uniqueness of solution for a periodic boundary value problem. Lakshmikantham and Círić[12 introduced the concept of mixed $g$-monotone mapping and proved coupled coincidence and coupled common fixed point theorems for commuting mappings, extending the theorems due to Bhaskar and Lakshmikantham [3]. Recently, Círić and Lakshmikantham [4] studied coupled random coincidence and coupled random fixed point theorems for a pair of random mappings $F: \Omega \times X \times X \longrightarrow X$ and $g: \Omega \times X \longrightarrow X$, where $X$ is a complete separable metric space and $(\Omega, \Sigma)$ is a measurable space, under some contractive conditions. Very recently, V. Berinde, M. Borcut [2] introduced the concept of tripled fixed point and proved some related theorems. In a natural fashion, some scholars have got triple and higher dimensional coincidence and fixed point results [1], 2], [10], [17], [18], 21], [22].

Motivated and inspired by the above results, we prove quadruple random coincidence and quadruple random fixed point theorems in partially ordered metric spaces for a random self-mapping $g$ and a random sequence $\left\{T_{i}\right\}_{n \in N}$ that have some useful properties.

For simplicity, we denote $\underbrace{X \times X \times X \times \ldots \times X}_{k \text { times }}$ by $X^{k}$ where $k \in N$. Let us recall some basic definitions.

Let $(\Omega, \Sigma)$ be a measurable space with $\Sigma$ being a $\sigma$-algebra of subsets of $\Omega$, and let $(X, d)$ be a metric space. A mapping $T: \Omega \rightarrow X$ is called $\Sigma$-measurable, if for any open subset $U$ of $X, T^{-1}(U)=$ $\{\omega: T \omega \in U\} \in \Sigma$. In what follows, when we speak of measurability, we will mean $\Sigma$-measurability. A mapping $T: \Omega \times X \rightarrow X$ is called a random operator if for any $x \in X, T(\cdot, x)$ is measurable. A measurable mapping $\zeta: \Omega \rightarrow X$ is called a random fixed point of a random function $T: \Omega \times X \rightarrow X$ if $\zeta(\omega)=T(\omega, \zeta(\omega))$ for every $\omega \in \Omega$. A measurable mapping $\zeta: \Omega \times X \rightarrow X$ is called a random coincidence point of $T: \Omega \times X \rightarrow X$ and $g: \Omega \times X \rightarrow X$ if $g(\omega, \zeta(\omega))=T(\omega, \zeta(\omega))$ for every $\omega \in \Omega$.

Definition $1.1([6])$. Let $(X, \leq, d)$ be a complete separable partially ordered metric space and $(\Omega, \Sigma)$ be a measurable space. Then $F: \Omega \times(X \times X) \rightarrow X$ and $g: \Omega \times X \rightarrow X$ are said to be compatible random operators, if

$$
\lim _{n \rightarrow \infty} d\left(g\left(\omega, F\left(\omega,\left(x_{n}, y_{n}\right)\right)\right), F\left(\omega, g\left(\omega,\left(x_{n}, y_{n}\right)\right)\right)=0\right.
$$

and

$$
\lim _{n \rightarrow \infty} d\left(g\left(\omega, F\left(\omega,\left(y_{n}, x_{n}\right)\right)\right), F\left(\omega, g\left(\omega,\left(y_{n}, x_{n}\right)\right)\right)=0,\right.
$$

whenever $\left\{x_{n}\right\}$ and $\left\{y_{n}\right\}$ are sequences in $X$ such that $\lim _{n \rightarrow \infty} g\left(\omega, x_{n}\right)=\lim _{n \rightarrow \infty} F\left(\omega,\left(x_{n}, y_{n}\right)\right)=x$ and $\lim _{n \rightarrow \infty} g\left(\omega, y_{n}\right)=\lim _{n \rightarrow \infty} F\left(\omega,\left(y_{n}, x_{n}\right)\right)=y$ for all $\omega \in \Omega$ and for all $x, y \in X$ being satisfied.

Definition $1.2([18])$. Let $(X, \leq, d)$ be a complete partially ordered metric space. We say that $X$ is regular if the following conditions hold:

(i) if a non-decreasing sequence $\left\{x_{n}\right\} \longrightarrow x$, then $x_{n} \leq x$ for all $n \geq 0$;

(ii) if a non-increasing sequence $\left\{y_{n}\right\} \longrightarrow y$, then $y_{n} \geq y$ for all $n \geq 0$.

Definition $1.3([21])$. Let $\left\{a_{n}\right\}$ be a sequence of non-negative real numbers. We say that a series $\sum_{n=1}^{+\infty} a_{n}$ is an $\alpha$-series, if there exist $0<\alpha<1$ and $n_{\alpha} \in N$ such that $\sum_{i=1}^{k} a_{i} \leq \alpha k$ for each $k \geq n_{\alpha}$.

Remark 1.4 ([21]). Each convergent series of non-negative real terms is an $\alpha$-series. However, there are also divergent series that are $\alpha$-series. For example, $\sum_{n=1}^{+\infty} \frac{1}{n}$ is an $\alpha$-series.

\section{Main results}

Let $(X, d, \leq)$ be a separable partially ordered space, $(\Omega . \Sigma)$ be a measurable space, $g$ be a random self-mapping on $\mathrm{X}$ and $\left\{T_{i}\right\} \in N$ be a sequence of random mappings from $\Omega \times X^{4}$ into $\mathrm{X}$ such that 
$T_{i}\left(\Omega \times X^{4}\right) \subseteq g(\Omega \times X)$ and

$$
\begin{aligned}
& T_{i}(\omega, x, y, z, t) \leq T_{i+1}(\omega, u, v, r, s), \quad T_{i}(\omega, y, z, t, x) \geq T_{i+1}(\omega, v, r, s, u), \\
& T_{i}(\omega, z, t, x, y) \leq T_{i+1}(\omega, r, s, u, v)
\end{aligned} \quad \text { and } \quad T_{i}(\omega, t, x, y, z) \geq T_{i+1}(\omega, s, u, v, r)
$$

for all $x, y, z, t, u, v, r, s \in X, \omega \in \Omega, i \in N$ with $g(\omega, x) \leq g(\omega, u), g(\omega, y) \geq g(\omega, v), g(\omega, z) \leq g(\omega, r) \quad$ and $g(\omega, t) \geq g(\omega, s)$.

In view of the above considerations, we obtain Definition 2.1 .

Definition 2.1. Let $(X, \leq, d)$ be a complete separable partially ordered metric space, $(\Omega, \Sigma)$ be a measurable space, $\left\{T_{i}\right\}_{i \in N}$ and $g$ are said to be random compatible operators if

$$
\begin{aligned}
& \lim _{n \rightarrow+\infty} d\left(g\left(\omega, T_{n}\left(\omega, x_{n}, y_{n}, z_{n}, t_{n}\right)\right), T_{n}\left(\omega, g\left(\omega, x_{n}\right), g\left(\omega, y_{n}\right), g\left(\omega, z_{n}\right), g\left(\omega, t_{n}\right)\right)\right)=0, \\
& \lim _{n \rightarrow+\infty} d\left(g\left(\omega, T_{n}\left(\omega, y_{n}, z_{n}, t_{n}, x_{n}\right)\right), T_{n}\left(\omega, g\left(\omega, y_{n}\right), g\left(\omega, z_{n}\right), g\left(\omega, t_{n}\right), g\left(\omega, x_{n}\right)\right)\right)=0, \\
& \lim _{n \rightarrow+\infty} d\left(g\left(\omega, T_{n}\left(\omega, z_{n}, t_{n}, x_{n}, y_{n}\right)\right), T_{n}\left(\omega, g\left(\omega, z_{n}\right), g\left(\omega, t_{n}\right), g\left(\omega, x_{n}\right), g\left(\omega, y_{n}\right)\right)\right)=0
\end{aligned}
$$

and

$$
\lim _{n \rightarrow+\infty} d\left(g\left(\omega, T_{n}\left(\omega, t_{n}, x_{n}, y_{n}, z_{n}\right)\right), T_{n}\left(\omega, g\left(\omega, t_{n}\right), g\left(\omega, x_{n}\right), g\left(\omega, y_{n}\right), g\left(\omega, z_{n}\right)\right)\right)=0 .
$$

Whenever $\left\{x_{n}\right\},\left\{y_{n}\right\},\left\{z_{n}\right\}$ and $\left\{t_{n}\right\}$ are sequences in $X$, such that

$$
\begin{aligned}
& \lim _{n \rightarrow+\infty} T_{n}\left(\omega, x_{n}, y_{n}, z_{n}, t_{n}\right)=\lim _{n \rightarrow+\infty} g\left(\omega, x_{n+1}\right)=x, \\
& \lim _{n \rightarrow+\infty} T_{n}\left(\omega, y_{n}, z_{n}, t_{n}, x_{n}\right)=\lim _{n \rightarrow+\infty} g\left(\omega, y_{n+1}\right)=y, \\
& \lim _{n \rightarrow+\infty} T_{n}\left(\omega, z_{n}, t_{n}, x_{n}, x_{n}\right)=\lim _{n \rightarrow+\infty} g\left(\omega, z_{n+1}\right)=z
\end{aligned}
$$

and

$$
\lim _{n \rightarrow+\infty} T_{n}\left(\omega, t_{n}, x_{n}, y_{n}, t_{n}\right)=\lim _{n \rightarrow+\infty} g\left(\omega, t_{n+1}\right)=t
$$

for some $x, y, z, t \in X, \omega \in \Omega$.

Definition 2.2. $\left\{T_{i}\right\}_{i \in N}$ and $g$ are called random weakly reciprocally continuous if

$$
\begin{aligned}
& \lim _{n \rightarrow+\infty} g\left(\omega, T_{n}\left(\omega, x_{n}, y_{n}, z_{n}, t_{n}\right)\right)=g(\omega, x), \\
& \lim _{n \rightarrow+\infty} g\left(\omega, T_{n}\left(\omega, y_{n}, z_{n}, t_{n}, x_{n}\right)\right)=g(\omega, y), \\
& \lim _{n \rightarrow+\infty} g\left(\omega, T_{n}\left(\omega, z_{n}, t_{n}, x_{n}, y_{n}\right)\right)=g(\omega, z)
\end{aligned}
$$

and

$$
\lim _{n \rightarrow+\infty} g\left(\omega, T_{n}\left(\omega, t_{n}, x_{n}, y_{n}, z_{n}\right)\right)=g(\omega, t),
$$

whenever $\left\{x_{n}\right\},\left\{y_{n}\right\},\left\{z_{n}\right\}$ and $\left\{t_{n}\right\}$ are sequences in $X$, such that

$$
\begin{aligned}
& \lim _{n \rightarrow+\infty} T_{n}\left(\omega, x_{n}, y_{n}, z_{n}, t_{n}\right)=\lim _{n \rightarrow+\infty} g\left(\omega, x_{n+1}\right)=x, \\
& \lim _{n \rightarrow+\infty} T_{n}\left(\omega, y_{n}, z_{n}, t_{n}, x_{n}\right)=\lim _{n \rightarrow+\infty} g\left(\omega, y_{n+1}\right)=y, \\
& \lim _{n \rightarrow+\infty} T_{n}\left(\omega, z_{n}, t_{n}, x_{n}, x_{n}\right)=\lim _{n \rightarrow+\infty} g\left(\omega, z_{n+1}\right)=z
\end{aligned}
$$

and

$$
\lim _{n \rightarrow+\infty} T_{n}\left(\omega, t_{n}, x_{n}, y_{n}, t_{n}\right)=\lim _{n \rightarrow+\infty} g\left(\omega, t_{n+1}\right)=t
$$

for some $x, y, z, t \in X, \omega \in \Omega$. 
Theorem 2.3. Let $(X, \leq, d)$ be a separable partially ordered metric space, $(\Omega, \Sigma)$ be a measurable space. Let $g$ be a random self-mapping on $X$ and $\left\{T_{i}\right\}_{i \in N}$ be a sequence of random mappings from $\Omega \times X^{4}$ into $X$ such that $T_{i}\left(\Omega \times X^{4}\right) \subseteq g(\Omega \times X), g(\Omega \times X)$ is a complete subset of $X,\left\{T_{i}\right\}_{i \in N}$ and $g$ are compatible random operators, random weakly reciprocally continuous, $g$ is monotonic non-decreasing, continuous, satisfying condition (2.1) and the following condition:

$$
\begin{aligned}
d\left(T_{i}(\omega, x, y, z, t), T_{j}(\omega, u, v, r, s) \leq\right. & \beta_{i, j}\left[d\left(g(\omega, x), T_{i}(\omega, x, y, z, t)\right)+d\left(g(\omega, u), T_{j}(\omega, u, v, r, s)\right)\right] \\
& +\gamma_{i, j} d(g(\omega, u), g(\omega, x))
\end{aligned}
$$

for $x, y, z, t, u, v, r, s \in X$ with $g(\omega, x) \leq g(\omega, u), g(\omega, v) \leq g(\omega, y), g(\omega, z) \leq g(\omega, r), g(\omega, s) \leq g(\omega, t)$; $0 \leq \beta_{i, j}, \gamma_{i, j}<1$ for $i, j \in N$ and $\limsup _{n \rightarrow \infty} \beta_{i, n}<1$.

Suppose also that there exist measurable mappings $\xi_{0}, \eta_{0}, \zeta_{0}, \rho_{0}: \Omega \rightarrow X$ such that

$$
\begin{gathered}
g\left(\omega, \xi_{0}\right) \leq T_{0}\left(\omega, \xi_{0}, \eta_{0}, \zeta_{0}, \rho_{0}\right), \\
g\left(\omega, \eta_{0}\right) \geq T_{0}\left(\omega, \eta_{0}, \zeta_{0}, \rho_{0}, \xi_{0}\right), \\
g\left(\omega, \zeta_{0}\right) \leq T_{0}\left(\omega, \zeta_{0}, \rho_{0}, \xi_{0}, \eta_{0}\right)
\end{gathered}
$$

and

$$
g\left(\omega, \rho_{0}\right) \geq T_{0}\left(\omega, \rho_{0}, \xi_{0}, \eta_{0}, \zeta_{0}\right)
$$

for all $\omega \in \Omega$. If $\sum_{i=1}^{+\infty}\left(\frac{\beta_{i, i+1}+\gamma_{i, i+1}}{1-\beta_{i, i+1}}\right)$ is an $\alpha$-series and $g(\Omega \times X)$ is regular, then $\left\{T_{i}\right\}_{i \in N}$ and $g$ have a quadruple random coincidence point.

Proof. Let $\Theta=\{\zeta: \Omega \rightarrow X\}$ be a family of measurable mappings. Define a function $h: \Omega \times X \rightarrow R^{+}$as follows:

$$
h(\omega, x)=d(x, g(\omega, x)) .
$$

Since $x \rightarrow g(\omega, x)$ is continuous for all $\omega \in \Omega$, we conclude that $h(\omega, \cdot)$ is continuous for all $\omega \in \Omega$. Also, since $x \rightarrow g(\omega, x)$ is measurable for all $x \in X$, we conclude that $h(\cdot, x)$ is measurable for all $\omega \in \Omega([23]$, p.868). Thus, $h(\omega, x)$ is the Caratheodory function. Therefore, if $\zeta: \Omega \rightarrow X$ is a measurable mapping, then $\omega \rightarrow h(\omega, \zeta(\omega))$ is also measurable [19]. Also, for each $\zeta \in \Theta$, the function $\eta: \Omega \rightarrow X$ defined by $\eta(\omega)=g(\omega, \zeta(\omega))$ is measurable, that is, $\eta \in \Theta$.

Now, we shall construct four sequences of measurable mappings $\left\{\xi_{n}(\omega)\right\},\left\{\eta_{n}(\omega)\right\},\left\{\zeta_{n}(\omega)\right\}$ and $\left\{\rho_{n}(\omega)\right\}$ in $\Theta$, and four sequences $\left\{g\left(\omega, \xi_{n}(\omega)\right)\right\},\left\{g\left(\omega, \eta_{n}(\omega)\right)\right\},\left\{g\left(\omega, \zeta_{n}(\omega)\right)\right\}$ and $\left\{g\left(\omega, \rho_{n}(\omega)\right)\right\}$ in $X$ as follows. Let $\xi_{0}, \eta_{0}, \zeta_{0}, \rho_{0} \in \Theta$ be such that

$$
\begin{gathered}
g\left(\omega, \xi_{0}(\omega)\right) \leq T_{0}\left(\omega, \xi_{0}(\omega), \eta_{0}(\omega), \zeta_{0}(\omega), \rho_{0}(\omega)\right), \\
g\left(\omega, \eta_{0}(\omega)\right) \geq T_{0}\left(\omega, \eta_{0}(\omega), \zeta_{0}(\omega), \rho_{0}(\omega), \xi_{0}(\omega)\right), \\
g\left(\omega, \zeta_{0}(\omega)\right) \leq T_{0}\left(\omega, \zeta_{0}(\omega), \rho_{0}(\omega), \xi_{0}(\omega), \eta_{0}(\omega)\right)
\end{gathered}
$$

and

$$
g\left(\omega, \rho_{0}(\omega)\right) \geq T_{0}\left(\omega, \rho_{0}(\omega), \xi_{0}(\omega), \eta_{0}(\omega), \zeta_{0}(\omega)\right)
$$

for all $\omega \in \Omega$. Since $T_{i}\left(\Omega \times X^{4}\right) \subseteq g(\Omega \times X)$ by an appropriate Filippov measurable implicit function theorem ([5], 8], [16]), we can choose $\xi_{0}, \eta_{0}, \zeta_{0}$ and $\rho_{0} \in \Theta$ such that

$$
\begin{aligned}
& g\left(\omega, \xi_{1}(\omega)\right)=T_{0}\left(\omega, \xi_{0}(\omega), \eta_{0}(\omega), \zeta_{0}(\omega), \rho_{0}(\omega)\right), \\
& g\left(\omega, \eta_{1}(\omega)\right)=T_{0}\left(\omega, \eta_{0}(\omega), \zeta_{0}(\omega), \rho_{0}(\omega), \xi_{0}(\omega)\right), \\
& g\left(\omega, \zeta_{1}(\omega)\right)=T_{0}\left(\omega, \zeta_{0}(\omega), \rho_{0}(\omega), \xi_{0}(\omega), \eta_{0}(\omega)\right), \\
& g\left(\omega, \rho_{1}(\omega)\right)=T_{0}\left(\omega, \rho_{0}(\omega), \xi_{0}(\omega), \eta_{0}(\omega), \zeta_{0}(\omega)\right),
\end{aligned}
$$


and for all $\omega \in \Omega$. Again taking into account that $T_{i}(\Omega \times X) \subseteq g(\Omega \times X)$ and continuing this process, we can construct sequence $\left\{\xi_{n}(\omega)\right\},\left\{\eta_{n}(\omega)\right\},\left\{\zeta_{n}(\omega)\right\}$ and $\left\{\rho_{n}(\omega)\right\}$ in $X$ such that

$$
\begin{aligned}
& g\left(\omega, \xi_{n+1}(\omega)\right)=T_{n}\left(\omega, \xi_{n}(\omega), \eta_{n}(\omega), \zeta_{n}(\omega), \rho_{n}(\omega)\right), \\
& g\left(\omega, \eta_{n+1}(\omega)\right)=T_{n}\left(\omega, \eta_{n}(\omega), \zeta_{n}(\omega), \rho_{n}(\omega), \xi_{n}(\omega)\right), \\
& g\left(\omega, \zeta_{n+1}(\omega)\right)=T_{n}\left(\omega, \zeta_{n}(\omega), \rho_{0}(\omega), \xi_{n}(\omega), \eta_{n}(\omega)\right), \\
& g\left(\omega, \rho_{n+1}(\omega)\right)=T_{n}\left(\omega, \rho_{n}(\omega), \xi_{n}(\omega), \eta_{n}(\omega), \zeta_{n}(\omega)\right)
\end{aligned}
$$

for all $\omega \in \Omega, n \geq 0$.

Now, by using mathematical induction, we prove that

$$
\begin{aligned}
& g\left(\omega, \xi_{n}(\omega)\right) \leq g\left(\omega, \xi_{n+1}(\omega)\right), \\
& g\left(\omega, \eta_{n}(\omega)\right) \geq g\left(\omega, \eta_{n+1}(\omega)\right), \\
& g\left(\omega, \zeta_{n}(\omega)\right) \leq g\left(\omega, \zeta_{n+1}(\omega)\right), \\
& g\left(\omega, \rho_{n}(\omega)\right) \geq g\left(\omega, \rho_{n+1}(\omega)\right)
\end{aligned}
$$

for all $n \geq 0$. Since

$$
\begin{gathered}
g\left(\omega, \xi_{0}(\omega)\right) \leq T_{0}\left(\omega, \xi_{0}(\omega), \eta_{0}(\omega), \zeta_{0}(\omega), \rho_{0}(\omega)\right), \\
g\left(\omega, \eta_{0}(\omega)\right) \geq T_{0}\left(\omega, \eta_{0}(\omega), \zeta_{0}(\omega), \rho_{0}(\omega), \xi_{0}(\omega)\right), \\
g\left(\omega, \zeta_{0}(\omega)\right) \leq T_{0}\left(\omega, \zeta_{0}(\omega), \rho_{0}(\omega), \xi_{0}(\omega), \eta_{0}(\omega)\right)
\end{gathered}
$$

and

$$
g\left(\omega, \rho_{0}(\omega)\right) \geq T_{0}\left(\omega, \rho_{0}(\omega), \xi_{0}(\omega), \eta_{0}(\omega), \zeta_{0}(\omega)\right),
$$

in view of 2.1 and (2.4), we have

$$
\begin{gathered}
g\left(\omega, \xi_{0}(\omega)\right) \leq g\left(\omega, \xi_{1}(\omega)\right), \\
g\left(\omega, \eta_{0}(\omega)\right) \geq g\left(\omega, \eta_{1}(\omega)\right), \\
g\left(\omega, \zeta_{0}(\omega)\right) \leq g\left(\omega, \zeta_{1}(\omega)\right)
\end{gathered}
$$

and

$$
g\left(\omega, \rho_{0}(\omega)\right) \geq g\left(\omega, \rho_{1}(\omega)\right)
$$

that is, 2.5 hold for $n=0$. We presume that 2.5) holds for some $n>0$. Now, by (2.1) and (2.4), one deduces that

$$
\begin{aligned}
g\left(\omega, \xi_{n+1}(\omega)\right) & =T_{n}\left(\omega, \xi_{n}(\omega), \eta_{n}(\omega), \zeta_{n}(\omega), \rho_{n}(\omega)\right) \\
& \leq T_{n+1}\left(\omega, \xi_{n+1}(\omega), \eta_{n+1}(\omega), \zeta_{n+1}(\omega), \rho_{n+1}(\omega)\right)=g\left(\omega, \xi_{n+2}(\omega)\right), \\
g\left(\omega, \eta_{n+1}(\omega)\right) & =T_{n}\left(\omega, \eta_{n}(\omega), \zeta_{n}(\omega), \rho_{n}(\omega), \xi_{n}(\omega)\right) \\
& \geq T_{n+1}\left(\omega, \eta_{n+1}(\omega), \zeta_{n+1}(\omega), \rho_{n+1}(\omega), \xi_{n+1}(\omega)\right)=g\left(\omega, \eta_{n+2}(\omega)\right), \\
g\left(\omega, \zeta_{n+1}(\omega)\right) & =T_{n}\left(\omega, \zeta_{n}(\omega), \rho_{n}(\omega), \xi_{n}(\omega), \eta_{n}(\omega)\right) \\
& \leq T_{n+1}\left(\omega, \zeta_{n+1}(\omega), \rho_{n+1}(\omega), \xi_{n+1}(\omega), \eta_{n+1}(\omega)\right)=g\left(\omega, \zeta_{n+2}(\omega)\right)
\end{aligned}
$$

and

$$
\begin{aligned}
g\left(\omega, \rho_{n+1}(\omega)\right) & =T_{n}\left(\omega, \rho_{n}(\omega), \xi_{n}(\omega), \eta_{n}(\omega), \zeta_{n}(\omega)\right) \\
& \geq T_{n+1}\left(\omega, \rho_{n+1}(\omega), \xi_{n+1}(\omega), \eta_{n+1}(\omega), \zeta_{n+1}(\omega)\right)=g\left(\omega, \rho_{n+2}(\omega)\right) .
\end{aligned}
$$

Thus by mathematical induction, we conclude that (2.5) holds for all $n \geq 0$. Therefore, we have

$$
\begin{aligned}
& g\left(\omega, \xi_{0}(\omega)\right) \leq g\left(\omega, \xi_{1}(\omega)\right) \leq g\left(\omega, \xi_{2}(\omega)\right) \leq \ldots \leq g\left(\omega, \xi_{n+1}(\omega)\right) \leq \ldots \\
& g\left(\omega, \eta_{0}(\omega)\right) \geq g\left(\omega, \xi_{1}(\omega)\right) \geq g\left(\omega, \eta_{2}(\omega)\right) \geq \ldots \geq g\left(\omega, \eta_{n+1}(\omega)\right) \geq \ldots
\end{aligned}
$$




$$
\begin{gathered}
g\left(\omega, \zeta_{0}(\omega)\right) \leq g\left(\omega, \zeta_{1}(\omega)\right) \leq g\left(\omega, \zeta_{2}(\omega)\right) \leq \ldots \leq g\left(\omega, \zeta_{n+1}(\omega)\right) \leq \ldots \\
g\left(\omega, \rho_{0}(\omega)\right) \geq g\left(\omega, \rho_{1}(\omega)\right) \geq g\left(\omega, \rho_{2}(\omega)\right) \geq \ldots \leq g\left(\omega, \rho_{n+1}(\omega)\right) \geq \cdots
\end{gathered}
$$

We denote

$$
\begin{aligned}
\delta_{n}= & d\left(g\left(\omega, \xi_{n}(\omega)\right), g\left(\omega, \xi_{n+1}(\omega)\right)\right)+d\left(g\left(\omega, \eta_{n}(\omega)\right), g\left(\omega, \eta_{n+1}(\omega)\right)\right) \\
& +d\left(g\left(\omega, \zeta_{n}(\omega)\right), g\left(\omega, \zeta_{n+1}(\omega)\right)\right)+d\left(g\left(\omega, \rho_{n}(\omega)\right), g\left(\omega, \rho_{n+1}(\omega)\right)\right) .
\end{aligned}
$$

Then by (2.2), we get

$$
\begin{aligned}
d\left(g\left(\omega, \xi_{1}(\omega)\right), g\left(\omega, \xi_{2}(\omega)\right)\right)= & d\left(T_{0}\left(\omega, \xi_{0}(\omega), \eta_{0}(\omega), \zeta_{0}(\omega), \rho_{0}(\omega)\right), T_{1}\left(\omega, \xi_{1}(\omega), \eta_{1}(\omega), \zeta_{1}(\omega), \rho_{1}(\omega)\right)\right) \\
\leq & \beta_{0,1}\left[d\left(g\left(\omega, \xi_{0}(\omega)\right), T_{0}\left(\omega, \xi_{0}, \eta_{0}(\omega), \zeta_{0}(\omega), \rho_{0}(\omega)\right)\right)\right. \\
& \left.+d\left(g\left(\omega, \xi_{1}(\omega)\right), T_{1}\left(\omega, \xi_{1}(\omega), \eta_{1}(\omega), \zeta_{1}(\omega), \rho_{1}(\omega)\right)\right)\right] \\
& +\gamma_{0,1} d\left(g\left(\omega, \xi_{0}(\omega)\right), g\left(\omega, \xi_{1}(\omega)\right)\right) \\
= & \beta_{0,1}\left[d\left(g\left(\omega, \xi_{0}(\omega)\right), g\left(\omega, \xi_{1}(\omega)\right)\right)+d\left(g\left(\omega, \xi_{1}(\omega)\right), g\left(\omega, \xi_{2}(\omega)\right)\right)\right] \\
& +\gamma_{0,1} d\left(g\left(\omega, \xi_{0}(\omega)\right), g\left(\omega, \xi_{1}(\omega)\right)\right) .
\end{aligned}
$$

It follows that

$$
\left(1-\beta_{0,1}\right) d\left(g\left(\omega, \xi_{1}(\omega)\right), g\left(\omega, \xi_{2}(\omega)\right)\right) \leq\left(\beta_{0,1}+\gamma_{0,1}\right) d\left(g\left(\omega, \xi_{0}(\omega)\right), g\left(\omega, \xi_{1}(\omega)\right)\right),
$$

or, equivalently,

$$
d\left(g\left(\omega, \xi_{1}(\omega)\right), g\left(\omega, \xi_{2}(\omega)\right)\right) \leq\left(\frac{\beta_{0,1}+\gamma_{0,1}}{1-\beta_{0,1}}\right) d\left(g\left(\omega, \xi_{0}(\omega)\right), g\left(\omega, \xi_{1}(\omega)\right)\right) .
$$

Also, one obtains

$$
\begin{aligned}
d\left(g\left(\omega, \xi_{2}(\omega)\right), g\left(\omega, \xi_{3}(\omega)\right)\right) & =d\left(T_{1}\left(\omega, \xi_{1}(\omega), \eta_{1}(\omega), \zeta_{1}(\omega), \rho_{1}(\omega)\right), T_{2}\left(\omega, \xi_{2}(\omega), \eta_{2}(\omega), \zeta_{2}(\omega), \rho_{2}(\omega)\right)\right) \\
& \leq\left(\frac{\beta_{1,2}+\gamma_{1,2}}{1-\beta_{1,2}}\right) d\left(g\left(\omega, \xi_{1}(\omega)\right), g\left(\omega, \xi_{2}(\omega)\right)\right) \\
& \leq\left(\frac{\beta_{0,1}+\gamma_{0,1}}{1-\beta_{0,1}}\right)\left(\frac{\beta_{1,2}+\gamma_{1,2}}{1-\beta_{1,2}}\right) d\left(g\left(\omega, \xi_{0}(\omega)\right), g\left(\omega, \xi_{1}(\omega)\right)\right) .
\end{aligned}
$$

Repeating the above procedure, we have

$$
d\left(g\left(\omega, \xi_{n}(\omega)\right), g\left(\omega, \xi_{n+1}(\omega)\right)\right) \leq \prod_{i=0}^{n-1}\left(\frac{\beta_{i, i+1}+\gamma_{i, i+1}}{1-\beta_{i, i+1}}\right) d\left(g\left(\omega, \xi_{0}(\omega)\right), g\left(\omega, \xi_{1}(\omega)\right)\right) .
$$

Using similar arguments as above, one can also show that

$$
\begin{aligned}
& d\left(g\left(\omega, \eta_{n}(\omega)\right), g\left(\omega, \eta_{n+1}(\omega)\right)\right) \leq \prod_{i=0}^{n-1}\left(\frac{\beta_{i, i+1}+\gamma_{i, i+1}}{1-\beta_{i, i+1}}\right) d\left(g\left(\omega, \eta_{0}(\omega)\right), g\left(\omega, \eta_{1}(\omega)\right)\right), \\
& d\left(g\left(\omega, \zeta_{n}(\omega)\right), g\left(\omega, \zeta_{n+1}(\omega)\right)\right) \leq \prod_{i=0}^{n-1}\left(\frac{\beta_{i, i+1}+\gamma_{i, i+1}}{1-\beta_{i, i+1}}\right) d\left(g\left(\omega, \zeta_{0}(\omega)\right), g\left(\omega, \zeta_{1}(\omega)\right)\right)
\end{aligned}
$$

and

$$
d\left(g\left(\omega, \rho_{n}(\omega)\right), g\left(\omega, \rho_{n+1}(\omega)\right)\right) \leq \prod_{i=0}^{n-1}\left(\frac{\beta_{i, i+1}+\gamma_{i, i+1}}{1-\beta_{i, i+1}}\right) d\left(g\left(\omega, \rho_{0}(\omega)\right), g\left(\omega, \rho_{1}(\omega)\right)\right) .
$$


From (2.6)-(2.9), we have

$$
\begin{aligned}
\delta_{n}= & d\left(g\left(\omega, \xi_{n}(\omega)\right), g\left(\omega, \xi_{n+1}(\omega)\right)\right)+d\left(g\left(\omega, \eta_{n}(\omega)\right), g\left(\omega, \eta_{n+1}(\omega)\right)\right) \\
& +d\left(g\left(\omega, \zeta_{n}(\omega)\right), g\left(\omega, \zeta_{n+1}(\omega)\right)\right)+d\left(g\left(\omega, \rho_{n}(\omega)\right), g\left(\omega, \rho_{n+1}(\omega)\right)\right) \\
\leq & \prod_{i=0}^{n-1}\left(\frac{\beta_{i, i+1}+\gamma_{i, i+1}}{1-\beta_{i, i+1}}\right)\left[d\left(g\left(\omega, \xi_{0}(\omega)\right), g\left(\omega, \xi_{1}(\omega)\right)\right)+d\left(g\left(\omega, \eta_{0}(\omega)\right), g\left(\omega, \eta_{1}(\omega)\right)\right)\right. \\
& \left.+d\left(g\left(\omega, \zeta_{0}(\omega)\right), g\left(\omega, \zeta_{1}(\omega)\right)\right)+d\left(g\left(\omega, \rho_{0}(\omega)\right), g\left(\omega, \rho_{1}(\omega)\right)\right)\right] \\
= & \prod_{i=0}^{n-1}\left(\frac{\beta_{i, i+1}+\gamma_{i, i+1}}{1-\beta_{i, i+1}}\right) \delta_{0} .
\end{aligned}
$$

Moreover, for $p>0$ and by repeated use of the triangle inequality, one obtains

$$
\begin{aligned}
d( & \left.g\left(\omega, \xi_{n}(\omega)\right), g\left(\omega, \xi_{n+p}(\omega)\right)\right)+d\left(g\left(\omega, \eta_{n}(\omega)\right), g\left(\omega, \eta_{n+p}(\omega)\right)\right)+d\left(g\left(\omega, \zeta_{n}(\omega)\right), g\left(\omega, \zeta_{n+p}(\omega)\right)\right) \\
& +d\left(g\left(\omega, \rho_{n}(\omega)\right), g\left(\omega, \rho_{n+p}(\omega)\right)\right) \\
\leq & d\left(g\left(\omega, \xi_{n}(\omega)\right), g\left(\omega, \xi_{n+1}(\omega)\right)\right)+d\left(g\left(\omega, \eta_{n}(\omega)\right), g\left(\omega, \eta_{n+1}(\omega)\right)\right)+d\left(g\left(\omega, \zeta_{n}(\omega)\right), g\left(\omega, \zeta_{n+1}(\omega)\right)\right) \\
& +d\left(g\left(\omega, \rho_{n}(\omega)\right), g\left(\omega, \rho_{n+1}(\omega)\right)\right) \\
& +d\left(g\left(\omega, \xi_{n+1}(\omega)\right), g\left(\omega, \xi_{n+2}(\omega)\right)\right)+d\left(g\left(\omega, \eta_{n+1}(\omega)\right), g\left(\omega, \eta_{n+2}(\omega)\right)\right)+d\left(g\left(\omega, \zeta_{n+1}(\omega)\right), g\left(\omega, \zeta_{n+2}(\omega)\right)\right) \\
& +d\left(g\left(\omega, \rho_{n+1}(\omega)\right), g\left(\omega, \rho_{n+2}(\omega)\right)\right)+\ldots \\
& +d\left(g\left(\omega, \xi_{n+p-1}(\omega)\right), g\left(\omega, \xi_{n+p}(\omega)\right)\right)+d\left(g\left(\omega, \eta_{n+p-1}(\omega)\right), g\left(\omega, \eta_{n+p}(\omega)\right)\right) \\
& +d\left(g\left(\omega, \zeta_{n+p-1}(\omega)\right), g\left(\omega, \zeta_{n+p}(\omega)\right)\right)+d\left(g\left(\omega, \rho_{n+p-1}(\omega)\right), g\left(\omega, \rho_{n+p}(\omega)\right)\right) \\
\leq & \prod_{i=0}^{n-1}\left(\frac{\beta_{i, i+1}+\gamma_{i, i+1}}{1-\beta_{i, i+1}}\right) \delta_{0}+\prod_{i=0}^{n}\left(\frac{\beta_{i, i+1}+\gamma_{i, i+1}}{1-\beta_{i, i+1}}\right) \delta_{0}+\ldots+\prod_{i=0}^{n+p-2}\left(\frac{\beta_{i, i+1}+\gamma_{i, i+1}}{1-\beta_{i, i+1}}\right) \delta_{0} \\
= & \sum_{k=0}^{p-1} \prod_{i=0}^{n+k-1}\left(\frac{\beta_{i, i+1}+\gamma_{i, i+1}}{1-\beta_{i, i+1}}\right) \delta_{0}=\sum_{k=n}^{n+p-1} \prod_{i=0}^{k-1}\left(\frac{\beta_{i, i+1}+\gamma_{i, i+1}}{1-\beta_{i, i+1}}\right) \delta_{0} .
\end{aligned}
$$

Since $\sum_{i=1}^{+\infty}\left(\frac{\beta_{i, i+1}+\gamma_{i, i+1}}{1-\beta_{i, i+1}}\right)$ is an $\alpha$-series and using the fact that the geometric mean of non-negative numbers is less than or equal to the arithmetic mean, it follows that

$$
\begin{aligned}
& d\left(g\left(\omega, \xi_{n}(\omega)\right), g\left(\omega, \xi_{n+p}(\omega)\right)\right)+d\left(g\left(\omega, \eta_{n}(\omega)\right), g\left(\omega, \eta_{n+p}(\omega)\right)\right)+d\left(g\left(\omega, \zeta_{n}(\omega)\right), g\left(\omega, \zeta_{n+p}(\omega)\right)\right) \\
& \quad+d\left(g\left(\omega, \rho_{n}(\omega)\right), g\left(\omega, \rho_{n+p}(\omega)\right)\right) \\
& \leq \sum_{k=n}^{n+p-1}\left[\frac{1}{k} \sum_{i=0}^{k-1}\left(\frac{\beta_{i, i+1}+\gamma_{i, i+1}}{1-\beta_{i, i+1}}\right)\right]^{k} \delta_{0} \\
& \leq\left(\sum_{k=n}^{n-p+1} \alpha^{k}\right) \delta_{0} \leq \frac{\alpha^{n}}{1-\alpha} \delta_{0} .
\end{aligned}
$$

Now, taking the limit as $n \rightarrow+\infty$, one deduces that

$$
\begin{aligned}
\lim _{n \rightarrow \infty}[ & \left(g\left(\omega, \xi_{n}(\omega)\right), g\left(\omega, \xi_{n+p}(\omega)\right)\right)+d\left(g\left(\omega, \eta_{n}(\omega)\right), g\left(\omega, \eta_{n+p}(\omega)\right)\right) \\
& \left.+d\left(g\left(\omega, \zeta_{n}(\omega)\right), g\left(\omega, \zeta_{n+p}(\omega)\right)\right)+d\left(g\left(\omega, \rho_{n}(\omega)\right), g\left(\omega, \rho_{n+p}(\omega)\right)\right)\right]=0 .
\end{aligned}
$$

Which further implies that

$$
\begin{aligned}
\lim _{n \rightarrow \infty} d\left(g\left(\omega, \xi_{n}(\omega)\right), g\left(\omega, \xi_{n+p}(\omega)\right)\right)=\lim _{n \rightarrow \infty} d\left(g\left(\omega, \eta_{n}(\omega)\right), g\left(\omega, \eta_{n+p}(\omega)\right)\right) \\
\left.\quad=\lim _{n \rightarrow \infty} d\left(g\left(\omega, \zeta_{n}(\omega)\right), g\left(\omega, \zeta_{n+p}(\omega)\right)\right)=\lim _{n \rightarrow \infty} d\left(g\left(\omega, \rho_{n}(\omega)\right), g\left(\omega, \rho_{n+p}(\omega)\right)\right)\right]=0 .
\end{aligned}
$$


Thus $\left\{g\left(\omega, \xi_{n}(\omega)\right)\right\},\left\{g\left(\omega, \eta_{n}(\omega)\right)\right\},\left\{g\left(\omega, \zeta_{n}(\omega)\right)\right\}$ and $\left\{g\left(\omega, \rho_{n}(\omega)\right)\right\}$ are Cauchy sequences in $X$. Since $g(\Omega \times X)$ is complete, then there exists $(\xi(\omega), \eta(\omega), \zeta(\omega), \rho(\omega)) \in \Theta$, such that

$$
\begin{aligned}
& \lim _{n \rightarrow+\infty} g\left(\omega, \xi_{n+1}(\omega)=\lim _{n \rightarrow \infty} T_{n}\left(\omega, \xi_{n}(\omega), \eta_{n}(\omega), \zeta_{n}(\omega), \rho_{n}(\omega)\right)=\xi(\omega),\right. \\
& \lim _{n \rightarrow+\infty} g\left(\omega, \eta_{n+1}(\omega)=\lim _{n \rightarrow \infty} T_{n}\left(\omega, \eta_{n}(\omega), \zeta_{n}(\omega), \rho_{n}(\omega), \xi_{n}(\omega)\right)=\eta(\omega),\right. \\
& \lim _{n \rightarrow+\infty} g\left(\omega, \zeta_{n+1}(\omega)=\lim _{n \rightarrow \infty} T_{n}\left(\omega, \zeta_{n}(\omega), \rho_{n}(\omega), \xi_{n}(\omega), \eta_{n}(\omega)\right)=\zeta(\omega)\right.
\end{aligned}
$$

and

$$
\lim _{n \rightarrow+\infty} g\left(\omega, \rho_{n+1}(\omega)=\lim _{n \rightarrow \infty} T_{n}\left(\omega, \rho_{n}(\omega), \xi_{n}(\omega), \eta_{n}(\omega), \zeta_{n}(\omega)\right)=\rho(\omega) .\right.
$$

Now, as $\left\{T_{i}\right\}_{i \in N}$ and $g$ are weakly reciprocally continuous, we have

$$
\begin{aligned}
& \lim _{n \rightarrow+\infty} g\left(\omega, T_{n}\left(\omega, \xi_{n}(\omega), \eta_{n}(\omega), \zeta_{n}(\omega), \rho_{n}(\omega)\right)\right)=g(\omega, \xi(\omega)), \\
& \lim _{n \rightarrow+\infty} g\left(\omega, T_{n}\left(\omega, \eta_{n}(\omega), \zeta_{n}(\omega), \rho_{n}(\omega), \xi_{n}(\omega)\right)\right)=g(\omega, \eta(\omega)), \\
& \lim _{n \rightarrow+\infty} g\left(\omega, T_{n}\left(\omega, \zeta_{n}(\omega), \rho_{n}(\omega), \xi_{n}(\omega), \eta_{n}(\omega)\right)\right)=g(\omega, \zeta(\omega))
\end{aligned}
$$

and

$$
\lim _{n \rightarrow+\infty} g\left(\omega, T_{n}\left(\omega, \rho_{n}(\omega), \xi_{n}(\omega), \eta_{n}(\omega), \zeta_{n}(\omega)\right)\right)=g(\omega, \rho(\omega)) .
$$

On the other hand, the compatibility of $\left\{T_{i}\right\}_{i \in N}$ and $g$ yields

$$
\begin{gathered}
\lim _{n \rightarrow+\infty} d\left(g \left(\omega, T_{n}\left(\omega, \xi_{n}(\omega), \eta_{n}(\omega), \zeta_{n}(\omega), \rho_{n}(\omega)\right), T_{n}\left(\omega, g\left(\omega, \xi_{n}(\omega), g\left(\omega, \eta_{n}(\omega), g\left(\omega, \zeta_{n}(\omega), g\left(\omega, \rho_{n}(\omega)\right)\right)=0,\right.\right.\right.\right.\right. \\
\lim _{n \rightarrow+\infty} d\left(g \left(\omega, T_{n}\left(\omega, \eta_{n}(\omega), \zeta_{n}(\omega), \rho_{n}(\omega), \xi_{n}(\omega)\right), T_{n}\left(\omega, g\left(\omega, \eta_{n}(\omega), g\left(\omega, \zeta_{n}(\omega), g\left(\omega, \rho_{n}(\omega), g\left(\omega, \xi_{n}(\omega)\right)\right)=0,\right.\right.\right.\right.\right. \\
\lim _{n \rightarrow+\infty} d\left(g \left(\omega, T_{n}\left(\omega, \zeta_{n}(\omega), \rho_{n}(\omega), \xi_{n}(\omega), \eta_{n}(\omega)\right), T_{n}\left(\omega, g\left(\omega, \zeta_{n}(\omega), g\left(\omega, \rho_{n}(\omega), g\left(\omega, \xi_{n}(\omega), g\left(\omega, \eta_{n}(\omega)\right)\right)=0\right.\right.\right.\right.\right.
\end{gathered}
$$
and

$\lim _{n \rightarrow+\infty} d\left(g\left(\omega, T_{n}\left(\omega, \rho_{n}(\omega), \xi_{n}(\omega), \eta_{n}(\omega), \zeta_{n}(\omega)\right), T_{n}\left(\omega, g\left(\omega, \rho_{n}(\omega), g\left(\omega, \xi_{n}(\omega), g\left(\omega, \eta_{n}(\omega), g\left(\omega, \zeta_{n}(\omega)\right)\right)=0\right.\right.\right.\right.\right.$.

Then we have

$$
\begin{gathered}
\lim _{n \rightarrow+\infty} T_{n}\left(\omega, g\left(\omega, \xi_{n}(\omega), g\left(\omega, \eta_{n}(\omega), g\left(\omega, \zeta_{n}(\omega), g\left(\omega, \rho_{n}(\omega)\right)=g(\omega, \xi(\omega)),\right.\right.\right.\right. \\
\lim _{n \rightarrow+\infty} T_{n}\left(\omega, g\left(\omega, \eta_{n}(\omega), g\left(\omega, \zeta_{n}(\omega), g\left(\omega, \rho_{n}(\omega), g\left(\omega, \xi_{n}(\omega)\right)=g(\omega, \eta(\omega)),\right.\right.\right.\right. \\
\lim _{n \rightarrow+\infty} T_{n}\left(\omega, g\left(\omega, \zeta_{n}(\omega), g\left(\omega, \rho_{n}(\omega), g\left(\omega, \xi_{n}(\omega), g\left(\omega, \eta_{n}(\omega)\right)=g(\omega, \zeta(\omega))\right.\right.\right.\right.
\end{gathered}
$$

and

$$
\lim _{n \rightarrow+\infty} T_{n}\left(\omega, g\left(\omega, \rho_{n}(\omega), g\left(\omega, \xi_{n}(\omega), g\left(\omega, \eta_{n}(\omega), g\left(\omega, \zeta_{n}(\omega)\right)=g(\omega, \rho(\omega)) .\right.\right.\right.\right.
$$

Since $\left\{g\left(\omega, \xi_{n}(\omega)\right)\right\}$ and $\left\{g\left(\omega, \zeta_{n}(\omega)\right\}\right.$ are non-decreasing and $\left\{g\left(\omega, \eta_{n}(\omega)\right)\right\}$ and $\left\{g\left(\omega, \rho_{n}(\omega)\right)\right\}$ is non-increasing, using the regularity of $X$, we have $g\left(\omega, \xi_{n}(\omega)\right) \leq \xi(\omega), \eta(\omega) \geq g\left(\omega, \eta_{n}(\omega)\right)$, $g\left(\omega, \zeta_{n}(\omega)\right) \leq \zeta(\omega)$ and $g\left(\omega, \rho_{n}(\omega)\right) \leq \rho(\omega)$ for all $n \geq 0$. Then by 2.2., one obtains

$$
\begin{aligned}
d\left(T_{i}(\omega, \xi(\omega), \eta(\omega), \zeta(\omega), \rho(\omega))\right. & T_{n}\left(\omega, \xi_{n}(\omega), \eta_{n}(\omega), \zeta_{n}(\omega), \rho_{n}(\omega)\right) \\
\leq & \beta_{i, n}\left[d\left(g(\omega, \xi(\omega)), T_{i}(\omega, \xi(\omega), \eta(\omega), \zeta(\omega), \rho(\omega))\right)\right. \\
& \left.\left.+d\left(g\left(\omega, \xi_{n}(\omega)\right)\right), T_{n}\left(\omega, g\left(\omega, \xi_{n}(\omega)\right), g\left(\omega, \eta_{n}(\omega)\right), g\left(\omega, \zeta_{n}(\omega)\right), g\left(\omega, \rho_{n}(\omega)\right)\right)\right)\right] \\
& \left.+\gamma_{i, n} d\left(g\left(\omega, \xi_{n}(\omega)\right)\right), g(\omega, \xi(\omega))\right) .
\end{aligned}
$$

Taking the limit as $n \rightarrow+\infty$, we obtain $T_{i}(\omega, \xi(\omega), \eta(\omega), \zeta(\omega), \rho(\omega))=g(\omega, \xi(\omega))$ as $\beta_{i, n}<1$. Similarly, it can be proved that $g(\omega, \eta(\omega))=T_{i}(\eta(\omega), \zeta(\omega), \rho(\omega), \xi(\omega)), g(\omega, \zeta(\omega))=T_{i}(\zeta(\omega), \rho(\omega), \xi(\omega), \eta(\omega))$ and $g(\omega, \rho(\omega))=T_{i}(\rho(\omega), \xi(\omega), \eta(\omega), \zeta(\omega))$. Thus, $(\xi(\omega), \eta(\omega), \zeta(\omega), \rho(\omega))$ is a quadruple coincidence point of $\left\{T_{i}\right\}_{i \in N}$ and $g$. 
If $g$ is the identity mapping, as a consequence of Theorem 2.3, we state the following corollary.

Corollary 2.4. Let $(X, d, \leq)$ be a complete separable partially ordered metric space. $(\Omega, \Sigma)$ be a measurable space. Let $\left\{T_{i}\right\}_{i \in N}$ be a sequence of random mappings from $\Omega \times X^{4}$ into $X$ such that $\left\{T_{i}\right\}_{i \in N}$ satisfies, for $x, y, z, t, u, v, r, s \in X$, with $x \leq u, v \geq y, z \leq r$ and $t \geq s$ the following conditions:

(i)

$$
\begin{gathered}
T_{i}(\omega, x, y, z, t) \leq T_{i+1}(\omega, u, v, r, s), \quad T_{i}(\omega, y, z, t, x) \geq T_{i+1}(\omega, v, r, s, u), \\
T_{i}(\omega, z, t, x, y) \leq T_{i+1}(\omega, r, s, u, v) \quad \text { and } \quad T_{i}(\omega, t, x, y, z) \geq T_{i+1}(\omega, s, u, v, r) .
\end{gathered}
$$

$$
\begin{aligned}
d\left(T_{i}(\omega, x, y, z, t), T_{j}(\omega, u, v, r, s)\right) & \leq \beta_{i, j}\left[d(\omega, x), T_{i}(\omega, x, y, z, t)+d(\omega, u), T_{j}(\omega, u, v, r, s)\right] \\
& +\gamma_{i, j} d((\omega, u),(\omega, x)),
\end{aligned}
$$

and $0 \leq \beta_{i, j}, \gamma_{i, j}<1$ for $i, j \in N$.

Suppose also that there exists measurable mappings $\xi_{0}, \eta_{0}, \zeta_{0}, \rho_{0}: \Omega \rightarrow X$ such that

$$
\begin{aligned}
\xi_{0}(\omega) & \leq T_{0}\left(\omega, \xi_{0}(\omega), \eta_{0}(\omega), \zeta_{0}(\omega), \rho_{0}(\omega)\right), \\
\eta_{0}(\omega) & \geq T_{0}\left(\omega, \eta_{0}(\omega), \zeta_{0}(\omega), \rho_{0}(\omega), \xi_{0}(\omega)\right), \\
\zeta_{0}(\omega) & \leq T_{0}\left(\omega, \zeta_{0}(\omega), \rho_{0}(\omega), \xi_{0}(\omega), \eta_{0}(\omega)\right)
\end{aligned}
$$

and

$$
\rho_{0}(\omega) \geq T_{0}\left(\omega, \rho_{0}(\omega), \xi_{0}(\omega), \eta_{0}(\omega), \zeta_{0}(\omega)\right)
$$

for all $\omega \in \Omega$. If $\sum_{i=1}^{+\infty}\left(\frac{\beta_{i, i+1}+\gamma_{i, i+1}}{1-\beta_{i, i+1}}\right)$ is an $\alpha$-series and $g(\Omega \times X)$ is regular, then $\left\{T_{i}\right\}_{i \in N}$ and $g$ have a random quadruple fixed point.

Now, we give useful conditions for the existence and uniqueness of a quadrupled common fixed point.

Theorem 2.5. In addition to the hypotheses of Theorem 2.3, suppose that for all measurable mappings $x, y, z, t: \Omega \rightarrow X$ and $u, v, r, s: \Omega \rightarrow X$, there exist measurable mappings $a, b, c, d: \Omega \rightarrow X$ such that

$$
\begin{aligned}
& \left(T_{i}(\omega, a(\omega), b(\omega), c(\omega), d(\omega)), T_{i}(\omega, b(\omega), c(\omega), d(\omega), a(\omega)),\right. \\
& \left.T_{i}(\omega, c(\omega), d(\omega), a(\omega), b(\omega)), T_{i}(\omega, d(\omega), a(\omega), b(\omega), c(\omega))\right)
\end{aligned}
$$

is comparable to

$$
\begin{aligned}
& \left(T_{i}(\omega, x(\omega), y(\omega), z(\omega), t(\omega)), T_{i}(\omega, y(\omega), z(\omega), t(\omega), x(\omega)),\right. \\
& \left.T_{i}(\omega, z(\omega), t(\omega), x(\omega), y(\omega)), T_{i}(\omega, t(\omega), x(\omega), y(\omega), z(\omega))\right), \\
& \left(T_{i}(\omega, u(\omega), v(\omega), r(\omega), s(\omega)), T_{i}(\omega, v(\omega), r(\omega), s(\omega), u(\omega)),\right. \\
& \left.T_{i}(\omega, r(\omega), s(\omega), u(\omega), v(\omega)), T_{i}(\omega, s(\omega), u(\omega), v(\omega), r(\omega))\right) .
\end{aligned}
$$

Then $\left\{T_{i}\right\}_{i \in N}$ and $g$ have a unique random quadruple common fixed point.

Proof. From Theorem 2.3, the set of quadruple random coincidence points is non-empty. Now, we show that if $(x(\omega), y(\omega), z(\omega), t(\omega))$ and $(u(\omega), v(\omega), r(\omega), s(\omega))$ are quadruple random coincidence points, that is, if

$$
\begin{aligned}
& g(\omega, x(\omega))=T_{i}(\omega, x(\omega), y(\omega), z(\omega), t(\omega)), \\
& g(\omega, y(\omega))=T_{i}(\omega, y(\omega), z(\omega), t(\omega), x(\omega)), \\
& g(\omega, z(\omega))=T_{i}(\omega, z(\omega), t(\omega), x(\omega), y(\omega)), \\
& g(\omega, t(\omega))=T_{i}(\omega, t(\omega), x(\omega), y(\omega), z(\omega)), \\
& g(\omega, u(\omega))=T_{i}(\omega, u(\omega), v(\omega), r(\omega), s(\omega)), \\
& g(\omega, v(\omega))=T_{i}(\omega, v(\omega), r(\omega), s(\omega), u(\omega)), \\
& g(\omega, r(\omega))=T_{i}(\omega, r(\omega), s(\omega), u(\omega), v(\omega)), \\
& g(\omega, s(\omega))=T_{i}(\omega, s(\omega), r(\omega), u(\omega), v(\omega)) .
\end{aligned}
$$


We prove that $(g(\omega, x(\omega)), g(\omega, y(\omega), g(\omega, z(\omega), g(\omega, t(\omega))$ and $(g(\omega, u(\omega), g(\omega, v(\omega), g(\omega, r(\omega), g(\omega, s(\omega))$ are equal. By assumption, there exists $\left(T_{i}(\omega, a(\omega), b(\omega), c(\omega), d(\omega))\right)$ is comparable to 2.12) and 2.13). Define sequences $\left\{g\left(\omega, a_{n}(\omega)\right)\right\},\left\{g\left(\omega, b_{n}(\omega)\right)\right\},\left\{g\left(\omega, c_{n}(\omega)\right)\right\}$ and $\left\{g\left(\omega, d_{n}(\omega)\right)\right\}$ such that $a(\omega)=a_{0}(\omega), b(\omega)=$ $b_{0}(\omega), c(\omega)=c_{0}(\omega)$ and $d(\omega)=d_{0}(\omega)$ for all $n$, and

$$
\begin{aligned}
& g\left(\omega, a_{n}(\omega)\right)=T_{n-1}\left(\omega, a_{n-1}(\omega), b_{n-1}(\omega), c_{n-1}(\omega), d_{n-1}(\omega)\right), \\
& g\left(\omega, b_{n}(\omega)\right)=T_{n-1}\left(\omega, b_{n-1}(\omega), c_{n-1}(\omega), d_{n-1}(\omega), a_{n-1}(\omega)\right), \\
& g\left(\omega, c_{n}(\omega)\right)=T_{n-1}\left(\omega, c_{n-1}(\omega), d_{n-1}(\omega), a_{n-1}(\omega), b_{n-1}(\omega)\right), \\
& g\left(\omega, d_{n}(\omega)\right)=T_{n-1}\left(\omega, d_{n-1}(\omega), a_{n-1}(\omega), b_{n-1}(\omega), c_{n-1}(\omega)\right) .
\end{aligned}
$$

Since (2.12) is comparable with (2.13), we can assume that $(g(\omega, x(\omega)), g(\omega, y(\omega)), g(\omega, z(\omega)), g(\omega, t(\omega))) \geq$ $(g(\omega, a(\omega)), g(\omega, b(\omega)), g(\omega, c(\omega)), g(\omega, d(\omega)))=\left(g\left(\omega, a_{0}(\omega)\right), g\left(\omega, b_{0}(\omega)\right), g\left(\omega, c_{0}(\omega)\right), g\left(\omega, d_{0}(\omega)\right)\right)$, then we get

$$
\begin{aligned}
d\left(g\left(\omega, x(\omega), g\left(\omega, a_{n+1}\right)\right)\right)= & d\left(T_{i}(\omega, x(\omega), y(\omega), z(\omega), t(\omega)), T_{n}\left(\omega, a_{n}(\omega), b_{n}(\omega), c_{n}(\omega), d_{n}(\omega)\right)\right) \\
\leq & \beta_{i, n}\left[d\left(g(\omega, x(\omega)), T_{i}(\omega, x(\omega), y(\omega), z(\omega), t(\omega))\right)\right. \\
& \left.+d\left(g\left(\omega, a_{n}(\omega)\right), T_{n}\left(\omega, a_{n}(\omega), b_{n}(\omega), c_{n}(\omega), d_{n}(\omega)\right)\right)\right] \\
& +\gamma_{i, n} d\left(g\left(\omega, x(\omega), g\left(\omega, a_{n}(\omega)\right)\right)\right) .
\end{aligned}
$$

Taking the limit as $n \rightarrow \infty$, we obtain $\lim _{n \rightarrow \infty} d\left(g\left(\omega, x(\omega), g\left(\omega, a_{n+1}\right)\right)\right)=0$ as $0<\beta_{i, n}, \gamma_{i, n}<1$. Similarly, it can be proved that

$$
\begin{array}{ll}
\lim _{n \rightarrow \infty} d\left(g\left(\omega, x(\omega), g\left(\omega, a_{n+1}\right)\right)\right)=0, & \lim _{n \rightarrow \infty} d\left(g\left(\omega, y(\omega), g\left(\omega, b_{n+1}\right)\right)\right)=0, \\
\lim _{n \rightarrow \infty} d\left(g\left(\omega, z(\omega), g\left(\omega, c_{n+1}\right)\right)\right)=0, & \lim _{n \rightarrow \infty} d\left(g\left(\omega, t(\omega), g\left(\omega, d_{n+1}\right)\right)\right)=0, \\
\lim _{n \rightarrow \infty} d\left(g\left(\omega, u(\omega), g\left(\omega, a_{n+1}\right)\right)\right)=0, & \lim _{n \rightarrow \infty} d\left(g\left(\omega, v(\omega), g\left(\omega, b_{n+1}\right)\right)\right)=0, \\
\lim _{n \rightarrow \infty} d\left(g\left(\omega, r(\omega), g\left(\omega, c_{n+1}\right)\right)\right)=0, & \lim _{n \rightarrow \infty} d\left(g\left(\omega, s(\omega), g\left(\omega, d_{n+1}\right)\right)\right)=0 .
\end{array}
$$

By compatibility of $T_{i}$ and $g$, we get

$$
\begin{aligned}
\lim _{n \rightarrow \infty} g\left(\omega, g\left(\omega, a_{n+1}(\omega)\right)\right) & =\lim _{n \rightarrow \infty} g\left(\omega, T_{n}\left(\omega, a_{n}(\omega), b_{n}(\omega), c_{n}(\omega), d_{n}(\omega)\right)\right) \\
& =\lim _{n \rightarrow \infty} T_{n}\left(\omega, g\left(\omega, a_{n}(\omega), g\left(\omega, b_{n}(\omega), g\left(\omega, c_{n}(\omega), g\left(\omega, d_{n}(\omega)\right)\right),\right.\right.\right. \\
\lim _{n \rightarrow \infty} g\left(\omega, g\left(\omega, b_{n+1}(\omega)\right)\right) & =\lim _{n \rightarrow \infty} g\left(\omega, T_{n}\left(\omega, b_{n}(\omega), c_{n}(\omega), d_{n}(\omega), a_{n}(\omega)\right)\right) \\
& =\lim _{n \rightarrow \infty} T_{n}\left(\omega, g\left(\omega, b_{n}(\omega), g\left(\omega, c_{n}(\omega), g\left(\omega, d_{n}(\omega), g\left(\omega, a_{n}(\omega)\right)\right),\right.\right.\right. \\
\lim _{n \rightarrow \infty} g\left(\omega, g\left(\omega, c_{n+1}(\omega)\right)\right) & =\lim _{n \rightarrow \infty} g\left(\omega, T_{n}\left(\omega, c_{n}(\omega), d_{n}(\omega), a_{n}(\omega), b_{n}(\omega)\right)\right) \\
& =\lim _{n \rightarrow \infty} T_{n}\left(\omega, g\left(\omega, c_{n}(\omega), g\left(\omega, d_{n}(\omega), g\left(\omega, a_{n}(\omega), g\left(\omega, b_{n}(\omega)\right)\right),\right.\right.\right. \\
\lim _{n \rightarrow \infty} g\left(\omega, g\left(\omega, d_{n+1}(\omega)\right)\right) & =\lim _{n \rightarrow \infty} g\left(\omega, T_{n}\left(\omega, d_{n}(\omega), a_{n}(\omega), b_{n}(\omega), c_{n}(\omega)\right)\right) \\
& =\lim _{n \rightarrow \infty} T_{n}\left(\omega, g\left(\omega, d_{n}(\omega), g\left(\omega, a_{n}(\omega), g\left(\omega, b_{n}(\omega), g\left(\omega, c_{n}(\omega)\right)\right) .\right.\right.\right.
\end{aligned}
$$

Set

$$
\lim _{n \rightarrow \infty} g\left(\omega, a_{n}(\omega)\right)=\xi(\omega), \quad \lim _{n \rightarrow \infty} g\left(\omega, b_{n}(\omega)\right)=\eta(\omega)
$$




$$
\lim _{n \rightarrow \infty} g\left(\omega, c_{n}(\omega)\right)=\zeta(\omega), \quad \lim _{n \rightarrow \infty} g\left(\omega, d_{n}(\omega)\right)=\rho(\omega)
$$

Then, we have

$$
\begin{aligned}
& g(\omega, \xi(\omega))=T_{i}(\omega, \xi(\omega), \eta(\omega), \zeta(\omega), \rho(\omega)), \\
& g(\omega, \eta(\omega))=T_{i}(\omega, \eta(\omega), \zeta(\omega), \rho(\omega), \xi(\omega)), \\
& g(\omega, \zeta(\omega))=T_{i}(\omega, \zeta(\omega), \rho(\omega), \xi(\omega), \eta(\omega)), \\
& g(\omega, \rho(\omega))=T_{i}(\omega, \rho(\omega), \xi(\omega), \eta(\omega), \zeta(\omega)) .
\end{aligned}
$$

Thus, $(\xi(\omega), \eta(\omega), \zeta(\omega), \rho(\omega))$ is a quadruple common random coincidence point of $T_{i}$ and $g$. Putting

$$
(u(\omega), v(\omega), r(\omega), s(\omega))=(\xi(\omega), \eta(\omega), \zeta(\omega), \rho(\omega)),
$$

we have

$$
\begin{array}{ll}
g(\omega, u(\omega))=g(\omega, \xi(\omega)), & g(\omega, v(\omega))=g(\omega, \eta(\omega)), \\
g(\omega, r(\omega))=g(\omega, \zeta(\omega)), & g(\omega, s(\omega))=g(\omega, \rho(\omega)) .
\end{array}
$$

From the above results, we have

$$
\begin{aligned}
& g(\omega, \xi(\omega))=T_{i}(\omega, \xi(\omega), \eta(\omega), \zeta(\omega), \rho(\omega))=\xi(\omega), \\
& g(\omega, \eta(\omega))=T_{i}(\omega, \eta(\omega), \zeta(\omega), \rho(\omega), \xi(\omega))=\eta(\omega), \\
& g(\omega, \zeta(\omega))=T_{i}(\omega, \zeta(\omega), \rho(\omega), \xi(\omega), \eta(\omega))=\zeta(\omega), \\
& g(\omega, \rho(\omega))=T_{i}(\omega, \rho(\omega), \xi(\omega), \eta(\omega), \zeta(\omega))=\rho(\omega) .
\end{aligned}
$$

Thus, $(\xi(\omega), \eta(\omega), \zeta(\omega), \rho(\omega))$ is a unique quadruple random fixed point.

\section{An application}

In this section, we give an example to demonstrate Theorem 2.3 .

Example 3.1. Let $X=[0,1]$, define $d(x, y)=|x-y|, \Omega=[0,1]$ and let $\Sigma$ be the sigma algebra of Lebesgue's measurable subset of [0,1]. Define $g: \Omega \times X \rightarrow X, T_{i}: \Omega \times X^{4} \rightarrow X$ and defines as $T_{i}(\omega, x, y, z, t)=\frac{(x+y+z+t) \omega}{4 i}, g(\omega, x)=\omega x$ for all $x, y, z, t \in X$ and $\omega \in \Omega$.

We check the sequences $\left\{x_{n}(\omega)\right\}=\frac{\omega}{n},\left\{y_{n}(\omega)\right\}=\frac{\omega}{n+1},\left\{z_{n}(\omega)\right\}=\frac{\omega}{n+2}$ and $\left\{t_{n}(\omega)\right\}=\frac{\omega}{n+3}$, one can easily observe that $\left\{T_{i}\right\}_{i \in N}$ and $g$ are random compatible, random weakly reciprocally continuous, $g$ is monotonic non-decreasing, continuous, as well as satisfying condition 2.2 .

By taking $0<\beta_{i, j}<1$ and $0 \leq \gamma_{i, j}<1$, we will check the inequality 2.2 is satisfied, thus all the hypotheses of Theorem 2.3 are satisfied and $(0,0,0,0),(1,1,1,1)$ are the quadruple random coincident points of $g$ and $T_{i}$. Moreover, using the same $T_{i}$ and $g$ in Theorem 2.5, $(0,0,0,0)$ is the unique quadruple random fixed point of $g$ and $T_{i}$.

\section{Acknowledgement}

The authors thank the editor and the referees for their valuable comments and suggestions. The research was supported by the National Natural Science Foundation of China $(11361042,11326099,11461045,11071108)$ and the Provincial Natural Science Foundation of Jiangxi, China (20132BAB201001, 20142BAB211016, 2010GZS0147).

\section{References}

[1] A. Alotaibi, S. Alsulami, Coupled coincidence points for monotone operators in partially ordered metric spaces, Fixed Point Theory Appl., 2011 (2011), 13 pages. 1

[2] V. Berinde, M. Borcut, Tripled fixed point theorems for contractive type mappings in partially ordered metric spaces, Nonlinear Anal., 74 (2011), 4889-4897 1 
[3] T. G. Bhaskar, V. Lakshmikantham, Fixed point theorems in partially ordered metric spaces and applications, Nonlinear Anal., 65 (2006), 1379-1393. 1

[4] L. Ćirić, V. Lakshmikantham, Coupled random fixed point theorems for nonlinear contractions in partially ordered metric spaces, Stoch. Anal. Appl., 27 (2009), 1246-1259. 1

[5] C. J. Himmelberg, Measurable relations, Fund. Math., 87 (1975), 53-72. 2

[6] N. Hussain, A. R. Khan, R. P. Agarwal, Krasnosel'skii and Ky Fan type fixed point theorems in ordered Banach spaces, J. Nonlinear Convex Anal., 11 (2010), 475-489. 1, 1.1.

[7] N. Hussain, A. Latif, N. Shafqat, Weak contractive inequalities and compatible mixed monotone random operators in ordered metric spaces, J. Inequal. Appl., 2012 (2012), 20 pages. 1

[8] S. Itoh, A random fixed point theorem for a multi-valued contraction mapping, Pac. J. Math., 68 (1977), 85-90. 1,2

[9] B. Jiang, S. Xu, L. Shi, Coupled coincidence points for mixed monotone random operators in partially ordered metric spaces, Abstr. Appl. Anal. 2014 (2014), 9 pages. 1

[10] E. Karapinar, N. V. Luong, Quadruple fixed point theorems for nonlinear contractions, Comput. Math. Appl., 64 (2012), 1839-1848. 1

[11] A. Khan, N. Hussain, Random coincidence point theorem in Frechet spaces with applications, Stochastic Anal. Appl., 22 (2004), 155-167. 1

[12] V. Lakshmikantham, L.Ćirić, Coupled fixed point theorems for nonlinear contractions in partially ordered metric spaces, Nonlinear Anal., 70 (2009), 4341-4349. 1

[13] G. Z. Li, H. Duan, On Random Fixed Point Theorems of Random Monotone Operators, Appl. Math. Lett., 18 (2005), 1019-1026. 1

[14] S. Li, X. Xiao, L. Li, J. Lv, Random Approximation with Weak Contraction Random Operators and a Random Fixed Point Theorem for Nonexpansive Random Self-mappings, J. Inequal. Appl., 2012 (2012), 7 pages. 1

[15] T. C. Lin, Random approximations and random fixed point theorems for non-self maps, Proc. Amer. Math. Soc., 103 (1988), 1129-1135. 1

[16] E. J. McShane, R. B. J. Warified, On Filippov's implicit functions lemma, Proc. Amer. Math. Soc., 18 (1967), 41-47. 2

[17] Z. Mustafa, H. Aydi, E. Karapinar, Mixed g-monotone property and quadruple fied point theorems in partially ordered metric spaces, Fixed Point Theory Appl., 2012 (2012), 19 pages. 1

[18] H. K. Nashine, B. Samet, Fixed Point Results for Mappings Satisfying $(\psi, \varphi)$-weakly Contractive Condition in Partially Ordered Metric Spaces, Nonlinear Anal., 74 (2010), 2201-2209. 1.1 .2

[19] R. T. Rockafellar, Measurable dependence of convex sets and functions in parameters, J. Math. Anal. Appl., 28 (1969), 4-25. 2

[20] M. Saha, N. Ganguly, Random Fixed Point Theorem on a Ćirić-type Contractive Mapping and Its Consequence, Fixed Point Theory Appl., 2012 (2012), 18 pages. 1

[21] V. Sihag, R. K. Vats, C. Vetro, A fixed point theorem in G-metric spaces via $\alpha$-series, Quaest. Math., 37 (2014), $1-6.1$, 1.3, 1.4

[22] R. K. Vats, K. Tas, V. Sihag, A. Kumar, Triple Fixed Point Theorems Via $\alpha$-series in Partially Ordered Metric Spaces, J. Inequal. Appl., 2014 (2014), 12 pages. 1

[23] D. H. Wagner, Survey of measurable selection theorems, SIAM J. Control Optim., 15 (1977), 859-903. 2

[24] C. Zhu, C. Chen, Calculations of random fixed point index, J. Math. Anal. Appl., 339 (2008), 839-844. 1

[25] C. Zhu, Z. Xu, Random Ambiguous Point of Random $K(\omega)$-set-contractive Operator, J. Math. Anal. Appl., 328 (2006), 2-6. 1

[26] X. H. Zhu, J. Z. Xiao, Random Periodic Point and Fixed Point Results for Random Monotone Mappings in Ordered Polish Spaces, Fixed Point Theory Appl., 2010 (2010), 13 pages. 1 